\title{
CFD simulation with multiphase flows in porous media and open mineral storage pile
}

\author{
S. Torno, J. Toraño, I. Diego, M. Menéndez, M. Gent \& J. Velasco \\ School of Mines, University of Oviedo, Spain
}

\begin{abstract}
In open storage piles in bulk solids port terminals, power stations and cement factories, not only the solid and porous barrier behaviour in front of the pile is important, but also the effect that porous and solid barriers produce when they are behind the pile.

Considering the dust propagation behind the pile, the solid barriers are more effective than the porous ones. But, the effect of the porous barriers on the wind velocity distribution, mainly in zones between the barriers and the piles should be taken into account, regarding the total dust emission to the atmosphere and its propagation.

In these studies, wind and dust concentration measurements in laboratory and field were carried out. A hot-wire anemometer (Velocicalc Plus (TSI)) was used in the wind study and two dust collectors (E-Sampler, Met One Instruments, Inc., Oregon, USA) were used to measure the dust concentration (Total Particle like PM10); a meteorological station (E-Sampler, Met One Instruments, Inc., Oregon, USA) attached to a PM10 collector was also employed.

All these measurements were used to adjust the 3D CFD computational model (Ansys CFX 10.0): In the wind case through a k-epsilon turbulence model and the dust case by Lagrangian method.

These adjusted models allow us to carry out several simulations combining the effect of solid and porous fences in front of and behind the pile, as well as pile shape modifications and behaviour analysis according to the dust emission from several special configurations and its relation to the wind gust preferential directions.
\end{abstract}

Keywords: particle emission, Computational Fluid Dynamics (CFD), open storage pile, air pollution. 


\section{Introduction}

The movement of minerals in open storage piles produces particle emissions to the atmosphere, which were studied to minimize their effect in the area more or less near the pile.

In previous research [1], the use of solid barriers in storage pile protection which reduces dust emission to $66 \%$ in the worst environmental and industrial conditions, was shown.

Nevertheless, the use of this solid barrier produces a high velocity vortex between the barrier and the pile, causing a greater dust emission to the windward side of the pile, [2-4].

The authors, based on their own experience and other research like [5] and [6], changed a solid barrier for a $30 \%$ porosity barrier, proving that the dust emission is reduced by $78 \%$.

The $30 \%$ porosity is obtained from research carried out by [7], who have determined from the "Particle Tracking Velocimitry" that a porosity of $30 \%$ is the more effective.

Once the barrier effect (solid and porous ) in front of the open storage pile is studied, the need of studying the barrier effect behind the pile arises, thus the pile is protected against the wind with a barrier in front of it and the emitted dust is collected with a barrier behind the pile.

In this paper, the barrier effectiveness, solid and porous, in each of the studied position, is shown.

The 3D Computational Fluid Dynamics or CFD code Ansys CFX 10.0 is used to carry out the simulations, through the K-epsilon turbulence model [8], which was adjusted by the measurement campaigns carried out in the Mine of Carbonar S.A. situated in Asturias (North of Spain).

\section{Experimental description}

\subsection{Introduction}

The scale tests were carried out in the installations of Carbonar, which are shown in fig. 1. The equipment consisted of a metalic cone of $52 \mathrm{~cm}$ in height (h) and $69 \mathrm{~cm}$ in diameter, a metalic ventilation pipe of $3 \mathrm{~m}$ in-length and $300 \mathrm{~mm}$ in diameter, which is connected to a $12 \mathrm{kw}$ fan, which generates the wind flow.

Two barriers are added to these elements, which are placed in front of and behind the cone.

\subsection{Barrier in front of the cone}

In this case, the barrier has $52 \mathrm{~cm}$ in height and $1.78 \mathrm{~m}$ in length such as it was explained in [1] for the solid barrier, and it is placed at $25 \mathrm{~cm}$ from both the pipe and the cone.

In fig. 2 distribution of the elements used in the scale tests for the porous barrier, is shown. 


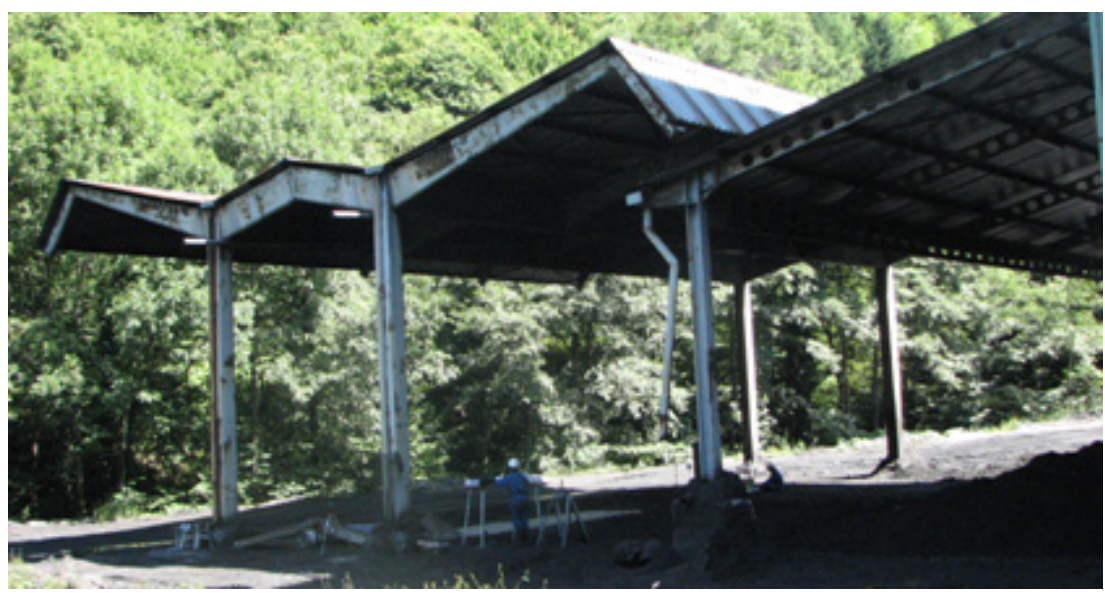

Figure 1: Zone where the equipment was installed.

\subsection{Barriers behind the cone.}

In this case the barrier is placed at $3.5 \mathrm{~m}$ behind the cone and it has $1 \mathrm{~m}$ in height and $2.04 \mathrm{~m}$ in length.

In fig. 3 the distribution of the elements for measurements in field with the dust collectors at $2.5 \mathrm{~m}$ behind the barrier, is shown.

\subsection{Velocity and concentration measurements.}

Flow velocity measurements were carried out with a hot-wire anemometer (Velocicalc Plus (TSI)), which was also used by other authors like [9] and [10], in 73 measurement points distributed in the cone zone and the barrier.

Two dust collector (E-Sampler, Met One Instruments, Inc) measuring total particles and PM10 ( $<10$ micron) were used to measure dust concentration. Meteorological conditions, relative humidity of atmosphere, temperature, wind velocity and the wind direction were measured by a meteorological station (ESampler, Met One Instruments, Inc) attached to a PM10 collector.

These field measurements were used to validate CFD simulation.

\section{Modelling}

The mathematical model is based on three equations: continuity, momentum and energy. These expressions form a partial differential equation system, coupled in linear form and they were used to achieve the solution.

The porosity input in the model is carried out from the research developed by [11] and [12] in which the absolute porosity value is calculated by two coefficients, the Linear Resistance Coefficient $\left(\mathrm{C}_{\mathrm{R} 1}\right)$ and the Quadratic Resistance Coefficient $\left(\mathrm{C}_{\mathrm{R} 2}\right)$ :

Determination of these coefficients is included in the discretisation of the Navier-Stokes equations. This discretisation produces a set of equations which 
describe the fluid properties, that is, momentum equation (1) and continuity equation (2):

$$
\begin{aligned}
& \frac{\partial \rho}{\partial \mathrm{t}}+\frac{\partial(\rho \mathrm{u})}{\partial \mathrm{x}}+\frac{\partial(\rho \mathrm{w})}{\partial \mathrm{z}}=0, \\
& \rho\left[\frac{\partial \mathrm{u}_{\mathrm{i}}}{\partial \mathrm{t}}+\mathrm{u}_{\mathrm{j}} \frac{\partial \mathrm{u}_{\mathrm{i}}}{\partial \mathrm{x}_{\mathrm{j}}}\right]=-\frac{\partial \mathrm{P}}{\partial \mathrm{x}_{\mathrm{i}}}+\frac{\partial}{\partial \mathrm{x}_{\mathrm{i}}}\left(\mu \frac{\partial^{2} \mathrm{u}_{\mathrm{i}}}{\partial \mathrm{x}_{\mathrm{j}} \partial \mathrm{x}_{\mathrm{j}}}\right)+\mathrm{S}_{\mathrm{Mi}} \text {. } \\
& \text { I II III IV }
\end{aligned}
$$

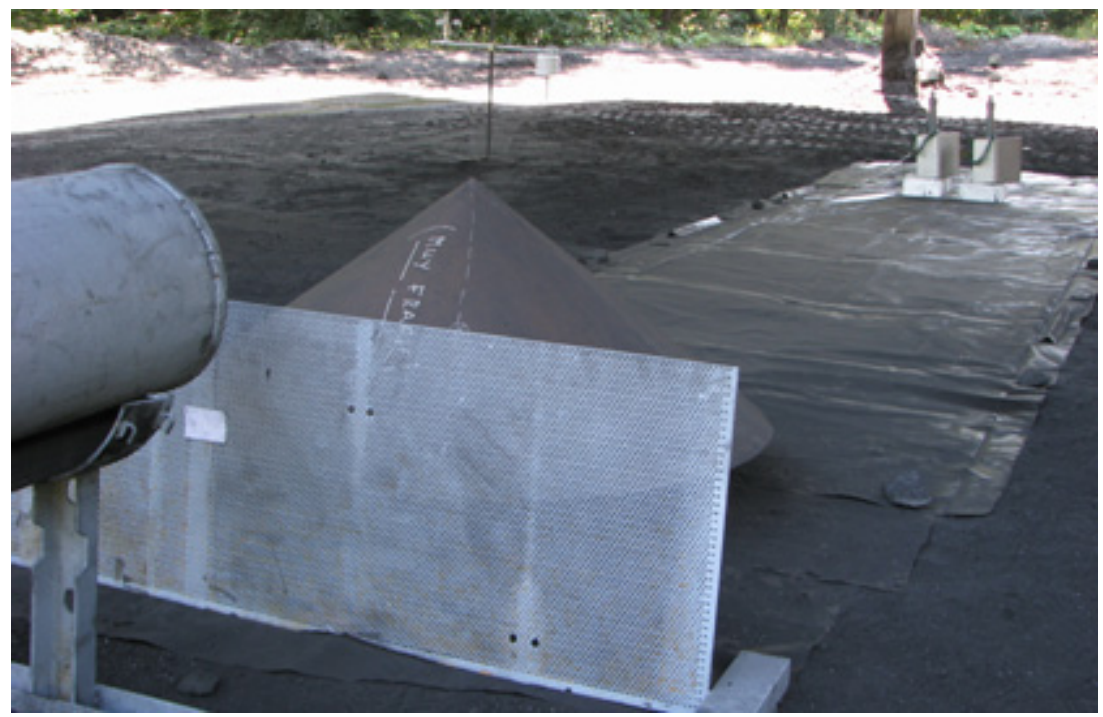

Figure 2: Distribution of the elements used for the porous barriers.

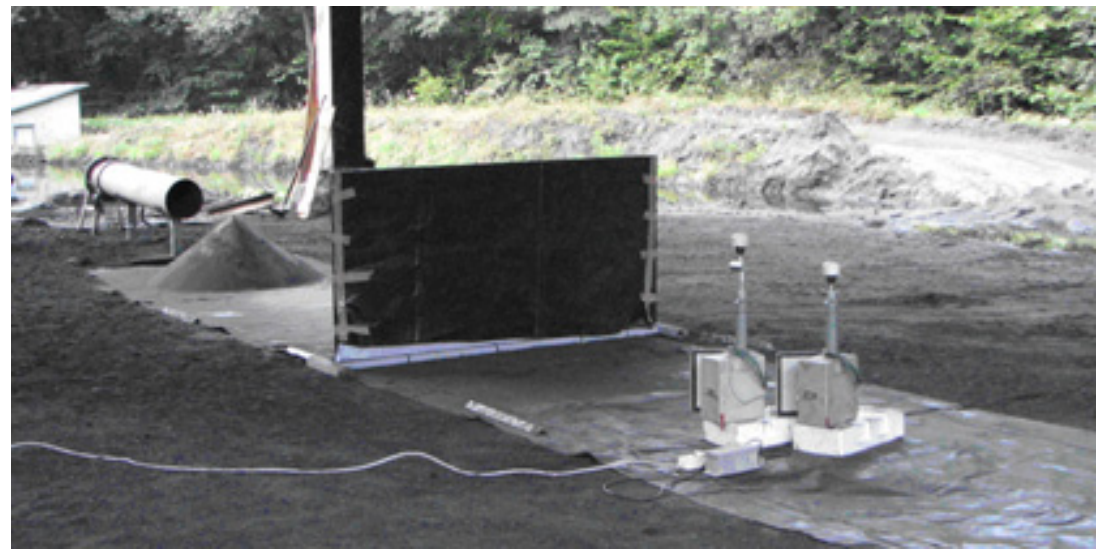

Figure 3: Distribution of the elements with the dust collectors. 
where, I is the Acceleration term, II is the Advection term, III is the Pressure term, IV is the Diffusion term and V is the Source term (3):

$$
\mathrm{S}_{\mathrm{Mi}}=-\mathrm{C}_{\mathrm{R} 1} \mathrm{u}_{\mathrm{i}}-\mathrm{C}_{\mathrm{R} 2}|\mathrm{U}| \mathrm{u}_{\mathrm{i}}
$$

where, $\mathrm{u}_{\mathrm{i}}$ is the velocity dimensional component along the 3 directions $(\mathrm{x}, \mathrm{y}, \mathrm{z}), \mathrm{U}$ is the velocity vector, $|\mathrm{U}|$ is the speed, $\mathrm{P}$ is pressure, $\rho$ is the density of incompressible air, $\mu$ is the dynamic viscosity and $S_{M i}$ are the source terms in the three directions; it indicates the porous barriers in the equation.

$\mathrm{C}^{\mathrm{R} 1}$ is set to $0\left(\mathrm{Kg} / \mathrm{m}^{3} \mathrm{~s}^{1}\right)$ assuming negligible viscous losses in the ambient air flow $\left(\mu=1.79 \times 10^{-5} \mathrm{~N} \mathrm{~s} / \mathrm{m}^{2}\right)$.

$\mathrm{C}^{\mathrm{R} 2}$ is set to $18.45\left(\mathrm{~kg} / \mathrm{m}^{4}\right)$, in a $30 \%$ porosity, [11].

These coefficients are related to absolute porosity via $\mathrm{K}_{\text {loss }}$ (empirical loss coefficient) $\left[\mathrm{C}_{\mathrm{R} 2} \cdot \varepsilon^{2} / \rho\right]$, which is included in Darcy's Law (4):

$$
-\frac{\partial \mathrm{P}}{\partial \mathrm{x}_{\mathrm{i}}}=\frac{\mu}{\mathrm{k}} \mathrm{u}_{\mathrm{i}}+\mathrm{k}_{\text {loss }} \rho|\mathrm{U}| \mathrm{u}_{\mathrm{i}}
$$

In fig. 4 , the geometry carried out by using SolidWorks for the barrier placed in front of the cone, is shown. The domain of the two models has the same dimensions $(8 \times 5 \times 25)$.

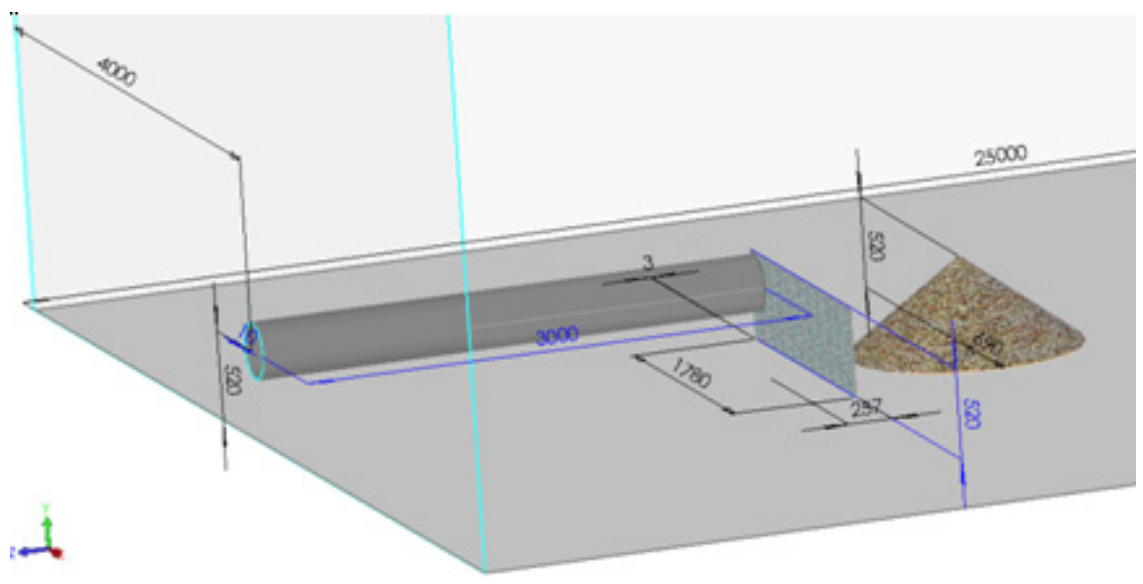

Figure 4: Geometry of the model with the barrier in front of the cone.

In fig. 5, the meshing carried out by using IcemCFD with the barrier placed behind the cone, is shown. This model was meshed with a total of 1,011,030 elements and the other model (barrier placed in front of the cone) was meshed with a total of $1,107,611$ elements.

In both cases it can be seen a finer meshing zone, where the dust collectors are situated, in which dust concentration measurements are taken for the simulations (fig. 5).

The fluid domain is a $25^{\circ}$ air affected by the gravity laws, not taking into account the heat transference and considering k-epsilon as the turbulence model. 
The boundary conditions have been an Inlet that corresponds to a $12 \mathrm{~m} / \mathrm{s}$ wind velocity coming out of a metallic pipe; the ground surface, cone, pipe and barrier as Wall and considering the rest as Opening.

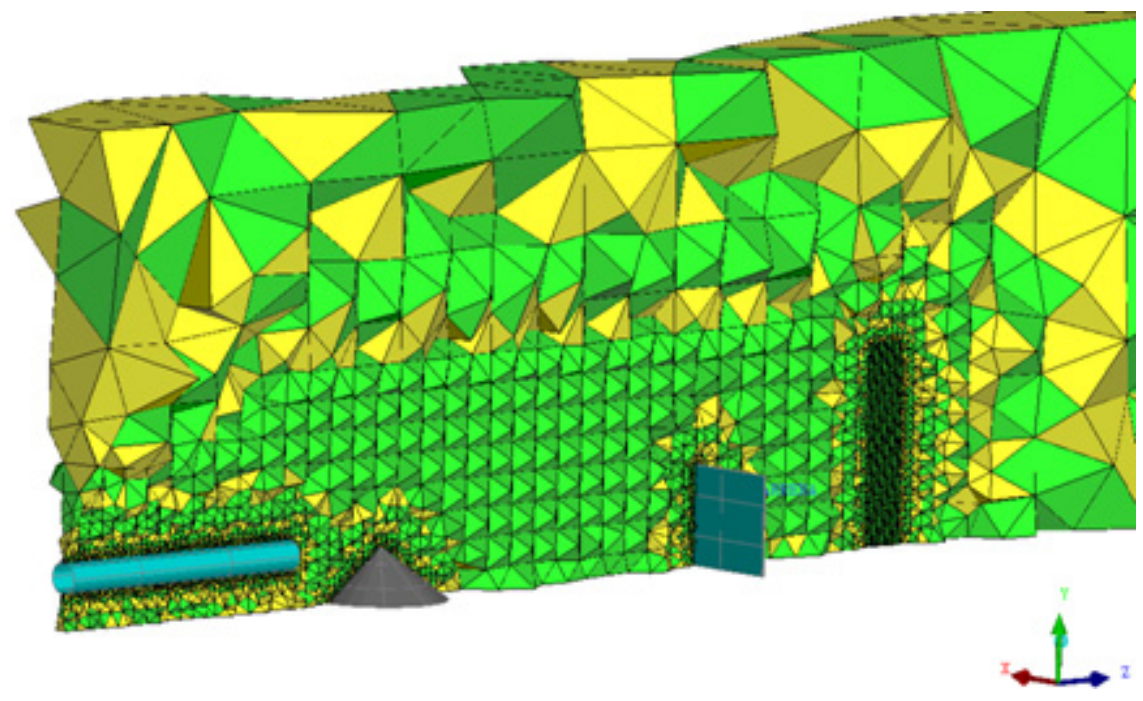

Figure 5: $\quad 3 \mathrm{D}$ meshing of the model with the barrier behind the cone.

\section{Wind results}

\subsection{Barriers in front of the cone}

Taking into account the results obtained in the model with a solid barrier (when porosity is $0 \%$ ), [1], it was decided to change the barrier porosity to $30 \%$.

This porous barrier makes the high velocity vortex that is produced behind the solid barrier, disappear, avoiding the dust set in suspension to windward zone of the cone.

In fig. 6 two planes of mean wind velocity for both cases, solid and porous barrier, are shown.

Besides, how the porous barrier eliminates the wind recirculation between the barrier and the cone, which is responsible for the dust emission on windward surface of the pile, is shown too.

These velocity values obtained from our simulations have been validated through velocity measurements in field for the same points.

In the solid barrier case, the equation that relates field measurements to those of the model is $y=0.9495 x-0.1191$ with a correlation coefficient $R^{2}=0.8859$. In the $30 \%$ porosity barrier case, the equation that relates field measurements to those of the model is $\mathrm{y}=1.5216 \mathrm{x}-1.2837$ with a correlation coefficient $\mathrm{R}^{2}=$ 0.8493 . The high values of the correlation coefficients indicate the suitability of the models. 


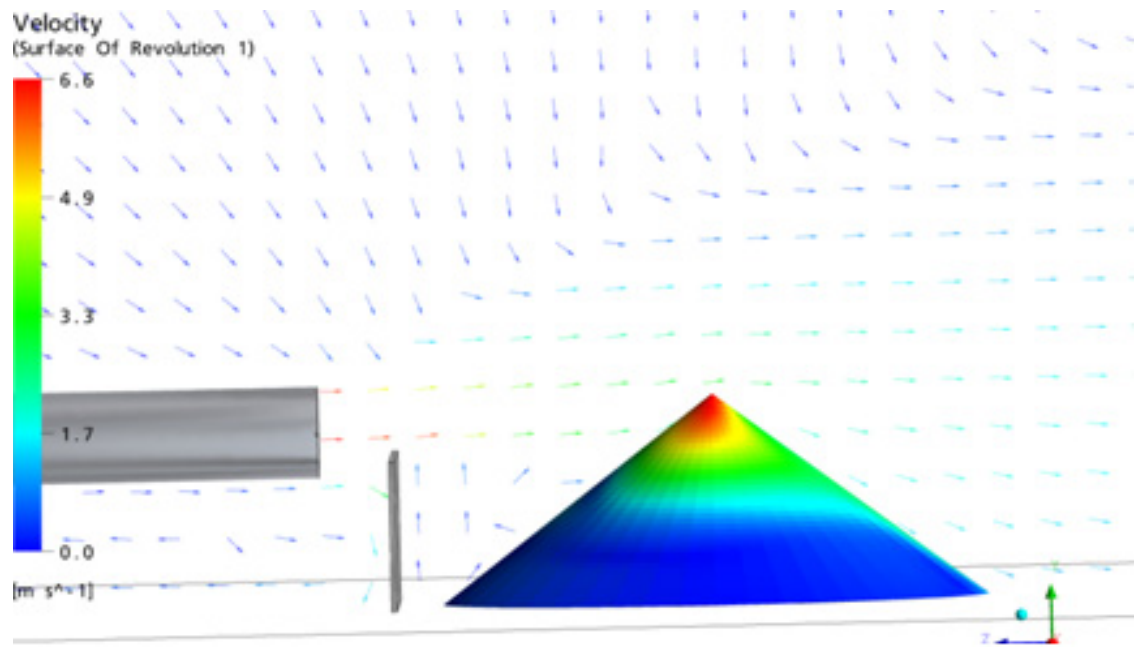

(a)

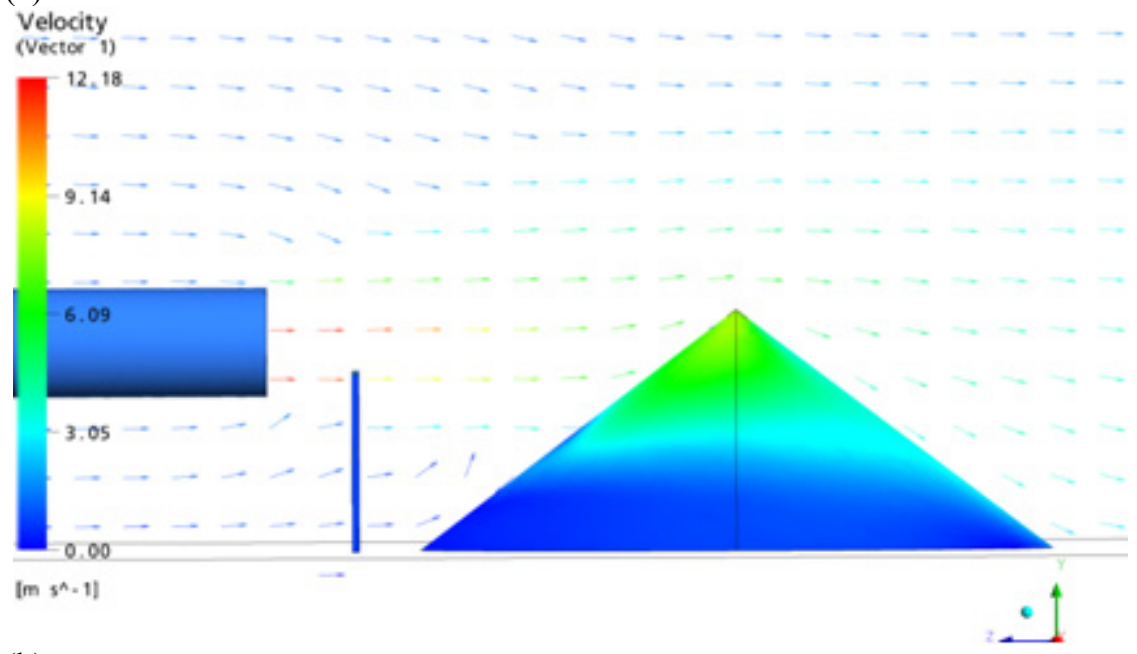

(b)

Figure 6: $\quad$ Planes of mean wind velocity for solid barrier (a) and porous barrier (b).

\subsection{Barriers behind the cone}

When the barrier is behind the cone, the solid barrier is the most effective, since it traps more quantity of dust emitted by the pile than the porous barrier.

In fig. 7 two planes of mean wind velocity perpendicular to the cone for solid and porous barrier, are shown.

It can be seen in the porous barrier case, how the air reaches the dust collectors with a higher velocity than in the solid barrier case. This is due to the recirculation zone of low wind velocity that is produced behind the solid barrier, 
As in the previous section, the velocity values obtained from our simulations have been validated through velocity measurements in field for the same points.

In the solid barrier case, the equation that relates field measurements to those of the model is $\mathrm{y}=0.9999 \mathrm{x}-0.2841$ with a correlation coefficient $\mathrm{R}^{2}=0.8756$. In the porous barrier case, the equation that relates field measurements to those of the model is $\mathrm{y}=2.5135 \mathrm{x}-3.9428$ with a correlation coefficient $\mathrm{R}^{2}=0.8463$.

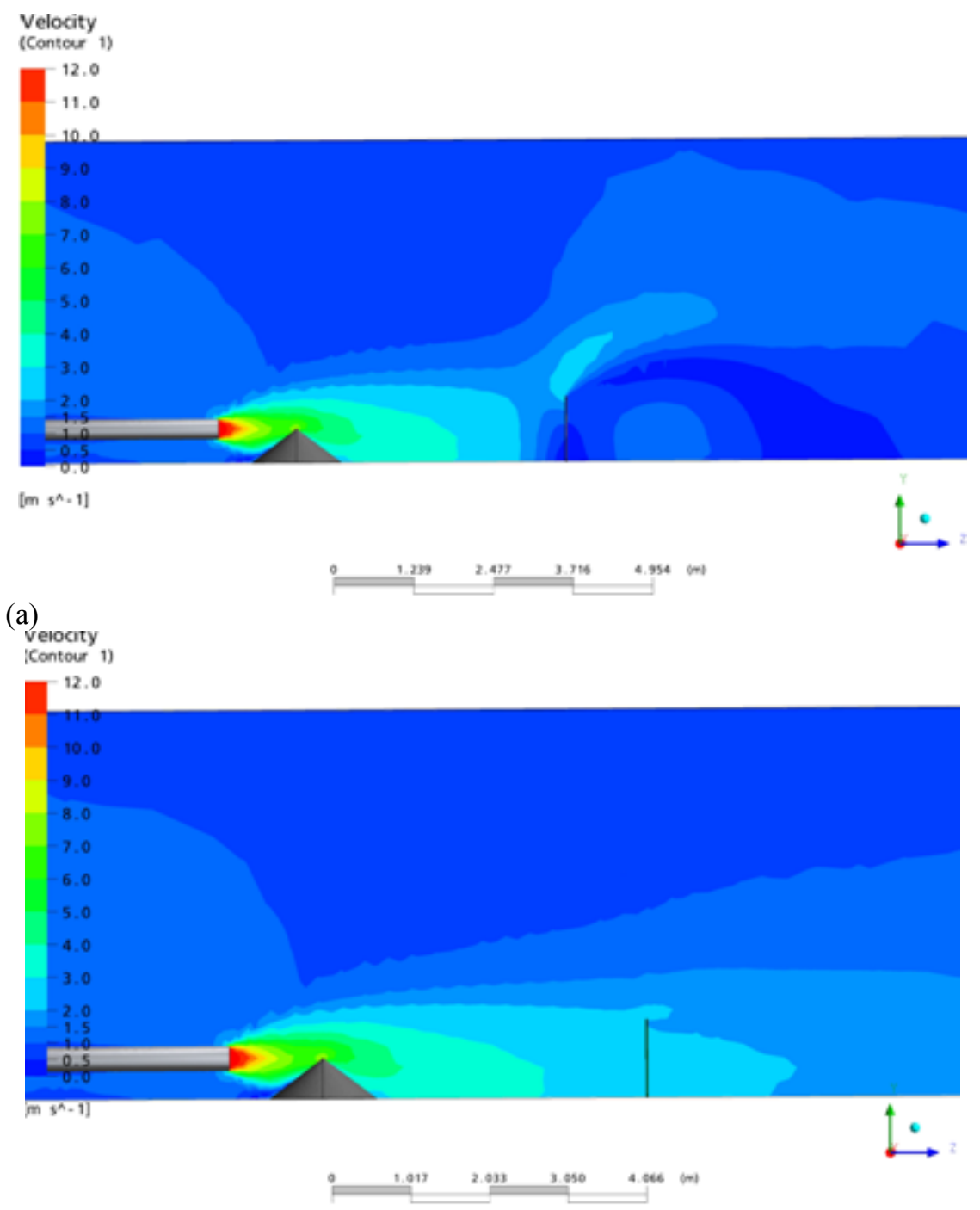

(b)

Figure 7: $\quad$ Planes of mean wind velocity for solid barrier (a) and porous barrier (b). 


\section{Concentration results}

Models are validated when field measurements coincide with simulations carried out by CFX.

The concentration value in software Ansys CFX is achieved through 'Particle Tracking', which is Lagrangian type solver that simulates particle trajectories from calculated velocity fields. This method starts from the continuous phase calculation (air) and they are used as input date for the dispersed phase (particles).

The millions of particles which are set in suspension in reality are simulated from the thousands of representative particles with a certain mass quantity; this is known as "Particle Number Rate" [8].

The concentration calculation is obtained from de calculation of the area under the Concentration-Time curve obtained by the PM10 dust collector.

This calculation is necessary as a permanent phenomenon (concentration by CFX) and a transitory phenomenon (concentration in field) are compared.

In table 1 the results of dust concentration obtained by modelling and those obtained in field for the two types of barriers, in front and behind the cone, are shown. The porous barrier placed in front of the cone presents less particle emission to the atmosphere.

The lower concentration value behind the cone corresponds to a solid barrier, as this barrier traps the most particles avoiding they spread farther. As it can be seen, in table 1 , the effect of porous barriers is to trap some particles and decrease the velocity of those crossing the barrier.

Table 1: Comparison between concentration measurements by CFX and in field.

\begin{tabular}{|l|l|l|l|l|}
\hline \multirow{2}{*}{$\begin{array}{l}\text { BARRIER } \\
\text { TYPE }\end{array}$} & \multicolumn{4}{l}{ CONCENTRATION $\left(\mathrm{mg} / \mathrm{m}^{3}\right)$} \\
\cline { 2 - 5 } & \multicolumn{2}{l}{ In front of the cone } & \multicolumn{2}{l|}{ Behind the cone } \\
\cline { 2 - 5 } & Experimental & Simulation & Experimental & Simulation \\
\hline Solid & 12.27 & 12.02 & 30.54 & 29.53 \\
\hline Porous & 7.86 & 8.124 & 34.05 & 34.97 \\
\hline
\end{tabular}

\section{Conclusion}

Ansys CFX software is a good tool to simulate the problems of dust set in suspension and its spreading in the surrounding area of an open storage pile.

When emissions of particulated material to the atmosphere are important it is necessary to use protection systems in front of the pile and behind it. These protection systems are solid and porous barriers and the effect they produce on dust emission from the pile depend on the position of this pile. 
The use of a porous barrier in front of the pile decreases the effect of the high velocity vortex which is produced behind the solid barrier, avoiding in this way the particle emission on windward surface of the pile. Behind the pile, a solid barrier traps more particles than a porous one.

\section{References}

[1] Diego, I., Toraño, J., Torno S. \& Garcia B., 2008. Experimental tests and Computational Fluid Dynamics (CFD) simulations of barriers installed around open storage piles of raw materials. Advances in Fluid Mechanics, 7, pp. 101-109.

[2] Lee, S.J. \& Lim, H.CH., 2001. A numerical study on ow around a triangular prism located behind a porous fence. Fluid Dynamics Research, 28, pp. 209-221.

[3] Toraño, J., Rodríguez, R., Diego, I., Rivas, J.M. \& Pelegry, A., 2007. Influence of the pile shape on wind erosion CFD emission simulation. Applied Mathematical Modelling, 31, pp. 2487-2502.

[4] Diego, I., Toraño, J., Torno, S. \& García, B., 2008. Experimental tests and Computational Fluid Dynamics (CFD) simulations of barriers installed around open storage piles of raw materials. Seventh International Conference on Advances in Fluid Mechanics (AFM 2008), The New Forest, UK.

[5] Lee, S.J. \& Kim, H.B., 1999. Laboratory measurements of velocity and turbulence field behind porous fences, Journal of Wind Engineering and Industrial Aerodynamics, pp. 311-326.

[6] Kim, H.B. \& Lee, S.J., 2001. Hole diameter effect on flow characteristics of wake behind porous fences having the same porosity. Fluid Dynamics Research, 28, pp. 449-464.

[7] Lee, S.J., Park, K.C. \& Park, C.W., 2002. Wind tunnel observations about the shelter effect of porous fences on the sand particle movements. Atmospheric Environment, 36, pp. 1453-1463.

[8] Diego, I., Pelegry, A., Torno, S., Toraño, J. \& Menendez M., 2009. Simultaneous CFD Evaluation of Wind Flow and Dust Emission in Open Storage Piles. Applied Mathematical Modelling. Article in press.

[9] Coleman, H.W., Steele, W.G, 1989. Experimentation and Uncertainty Analysis for Engineers. John Wiley \& Sons., Inc., New York.

[10] Kim, H.G., Lee, Ch.M., Lim, H.C. \& Kyong, N.H., 1997. An experimental and numerical study on the flow over two-dimensional hills. Journal of Wind Engineering and Industrial Aerodynamics, 66, pp. 17-33.

[11] Tiwary, A., Morvan, H.P. \& Colls, J.J., 2005. Modelling the size-dependent collection efficiency of hedgerows for ambient aerosols. Journal of Aerosol Science, 37, pp. 990-1015.

[12] Wang, H. \& Takle, E.S., 1995. A numerical simulation of boundary-layer flows near shelterbelts. Boundary-Layer Meteorology, 75, pp. 141-173.

[13] ANSYS CFX-Solver, Release 10.0: Theory, 2008. Flow in porous media, pp. 65-67. 